\title{
Clinical and Genetic Characterization of Huntington Disease among Hong Kong Chinese - A 5-year Review
}

\author{
Daniel Cheuk-Wa LEUNG ${ }^{1}$, Chloe Miu MAK ${ }^{1 *}$, Kam-Ming AU ${ }^{1}$, Wai-Kwan SIU ${ }^{1}$, Sammy Pak-Lam CHEN ${ }^{1}$, \\ Wing-Tat POON ${ }^{1}$, Shui-Fan TONG ${ }^{1}$, Yin-Chu SO0 ${ }^{1}$, Yuen-Wai LEE ${ }^{1}$, Chung-Leung CHAN ${ }^{1}$, Kwok-Wai YU ${ }^{1}$, \\ Suk-Yee LEE ${ }^{1}$, Wai-Shan HO ${ }^{1}$, Tin-Wing TONG ${ }^{1}$, Tsz-Kwan POON ${ }^{1}$, Tsz-Yan TONG ${ }^{1}$, Ho-Ying LEUNG ${ }^{1}$, Kwok- \\ kwong LAU ${ }^{2}$, Bun SHENG ${ }^{3}$ and Yan-wo CHAN ${ }^{1}$
}

${ }^{1}$ Kowloon West Cluster Laboratory Genetic Service, Chemical Pathology Laboratory, Department of Pathology, China

${ }^{2}$ Department of Medicine and Geriatrics, Princess Margaret Hospital, China

${ }^{3}$ Department of Pathology, The University of Hong Kong, China

Received: May 13, 2018; Published: May 22, 2018

*Corresponding author: Chloe Mak, 11/F, Block G, Kowloon West Cluster Laboratory Genetic Service, Chemical Pathology Laboratory, Department of Pathology, Princess Margaret Hospital, Hong Kong SAR, China

Abstract

Purpose: Huntington disease (HD) is a progressive and fatal autosomal dominant neurodegenerative disorder caused by trinucleotide CAG repeats in exon 1 of HTT. Incidence is lower among Chinese with limited data. We characterize the clinical and genetic data of six HD Hong Kong Chinese patients.

Materials and Methods: A total of 13 suspected cases were performed from 2008 to 2013. Repeat sizing was performed by PCR, agarose gel electrophoresis, fragment analysis by capillary electrophoresis and confirmed with Sanger sequencing. Triplet repeat primed PCR was performed for cases with one normal band detected. Reflex genetic testing of dentatorubral-pallidoluysian atrophy (DRPLA) was performed for all negative cases.

Results: Six were diagnosed of HD with CAG repeat > 40. Three positive cases had no HD family history. Two pre-symptomatic testing was performed with one positive. All symptomatic patients were adult onset with $43.4 \pm 11.6$ years (mean \pm SD; range 29-59 years). The duration from onset to genetic testing was $7 \pm 4.8$ years (mean $\pm \mathrm{SD}$; range $0-12$ years). The major clinical presentations were generalized chorea, behavioral disorders and dementia. The number of pathological expanded CAG repeats was $46 \pm 6.8$ (range $42-58$ ). None of those with negative HD mutation were positive for DRPLA.

Conclusion: We have characterized the clinical and genetic data of six Huntington disease Hong Kong Chinese patients. All were adult onset and had CAG repeat $>40$ and half had no HD family history. The duration from onset to genetic testing was significantly long. We have simplified the PCR protocols of triplet repeat primed PCR and Sanger sequencing without gel excision for expanded allele. We recommend reflex testing for other HD phenocopy conditions could be considered in patients with negative HD mutation.

Keywords: Hong Kong Chinese; Huntington disease; HTT

\section{Introduction}

Huntington Disease (HD, MIM \#143100) is an autosomal dominant neurological disease characterized with excessive motor movements and neuropsychological impairments. The earliest literature describing the symptoms related to this disease can be dated back to 1832 [1]. Not until 1872, an America physician, Dr. George Huntington published a medical literature which first characterized this disorder and formally named as HD [2]. However it was nearly a century later, in year 1993, a research group had worked up the genetic cause of HD which was due to an unstable trinucleotide repeats expansion on this disease chromosome within the interesting transcript number 15 (IT-15) which later named as Huntingtin (HTT, MIM*613004) gene [3]. HD is caused by cytosineadenosine-guanine (CAG) repeat expansions in HTT exon 1 on chromosome 4p16.3 resulting in expanded polyglutamine tracts.

Affected patients present with a collection of symptoms which include motor, cognitive and emotional disturbances. Clinical manifestations are gradually evolving involuntary movements and progressive dementia. Chorea is the most prominent abnormality. However, some can present with Parkinsonism, dystonia and ataxia with misdiagnosis such as spinocerebellar ataxias (SCA) [4-6]. 
HD phenocopies can be autosomal dominant, autosomal recessive or X-linked. Autosomal dominant examples are HD-like 2, HD-like 1, dentatorubropallidoluysian atrophy, SCA2, SCA17 (HD-like 4), C9orf72 mutations, benign hereditary chorea and neuroferritinopathy, whereas, autosomal recessive examples are Friedreich's ataxia, neuroacanthocytosis, ataxia telangiectasia, HD-like 3 and ataxia with oculomotor apraxia. McLeod syndrome is the X-linked HD mimic.

The sex incidence for HD is equal, with no sex differences in age of onset and clinical features. The average age of onset ranges from 35 to 50 years but varying from early childhood to 80 years old. In approximately $25 \%$ of individuals with HD, the onset is delayed after age 50 years, a few even after age 70 years. The course is relentlessly progressive with death occurs 15 - 20 years after disease onset. There is a negative association of number of CAG repeats with the age of disease onset. Juvenile onset HD has larger CAG repeats. Moreover, patients with paternal original have a greater repeat number than those with maternally transmitted. The phenomenon of anticipation, in which age of onset tends to decrease in successive generations; on average, affected children will develop the disease about 8 years earlier than their affected father. The clinical manifestation of anticipation can be dramatic; at times the children of HD-gene carriers will develop the disease before their parents. There is no curative treatment for HD at present. Predictive testing in asymptomatic adults at risk requires careful thought and genetic counseling. Pre-symptomatic testing on individuals younger than 18 years old is not recommended.

Table 1: Clinical and genetic findings of all referrals for HD genetic test.

\begin{tabular}{|c|c|c|c|c|c|c|}
\hline \multirow{2}{*}{ Case } & \multirow{2}{*}{$\begin{array}{l}\text { Sex / Age at } \\
\text { referral }(y)\end{array}$} & \multirow{2}{*}{ Clinical presentations } & \multirow{2}{*}{ Family history } & \multicolumn{2}{|c|}{ CAG repeat size } & \multirow{2}{*}{$\begin{array}{l}\text { Diagno- } \\
\text { sis }\end{array}$} \\
\hline & & & & Allele 1 & Allele 2 & \\
\hline 1 & $\mathrm{~F} / 51$ & $\begin{array}{l}\text { Chorea, pre-senile dementia, schizophrenia. Cerebral } \\
\text { atrophy }\end{array}$ & $\mathrm{N}$ & 16 & 21 & Normal \\
\hline 2 & $\mathrm{~F} / 45$ & Progressive choreoathetosis & $\mathrm{N}$ & 18 & 45 & HD \\
\hline 3 & $\mathrm{~F} / 60$ & $\begin{array}{c}\text { Choreoathetosis, ataxia and progressive cognitive } \\
\text { impairment. Cerebral atrophy }\end{array}$ & $\begin{array}{l}\text { Y } \\
\text { Father with abnormal movement and } \\
\text { was diagnosed as Parkinson's disease. }\end{array}$ & 17 & 43 & HD \\
\hline 4 & $\mathrm{~F} / 29$ & Unsteady gait and choreoathetosis & Y (Father) & 17 & 58 & HD \\
\hline 5 & $\mathrm{~F} / 52$ & Chorea, frequent falls and slurring speech & $\mathrm{N}$ & 17 & 42 & HD \\
\hline 6 & $\mathrm{~F} / 25$ & Asymptomatic & Y (42 CAG repeats in proband) & 17 & -- & Normal\# \\
\hline 7 & M / 64 & Dementia, chorea and aggressive behavior & $\mathrm{N}$ & 18 & -- & Normal\# \\
\hline 8 & $\mathrm{~F} / 55$ & $\begin{array}{l}\text { Progressive unsteady gait, dysarthria, chorea, slurring } \\
\text { speech }\end{array}$ & $\mathrm{N}$ & 17 & -- & Normal\# \\
\hline 9 & $\mathrm{~F} / 56$ & Chorea, cerebral atrophy & $\mathrm{N}$ & 17 & 18 & Normal \\
\hline 10 & $\mathrm{~F} / 59$ & Choreoathetosis and anxiety & Y (Two siblings) & 17 & 40 & HD \\
\hline 11 & $\mathrm{~F} / 37$ & Asymptomatic & Y (43 CAG repeats in proband) & 16 & 42 & HD \\
\hline 12 & $\mathrm{M} / 8$ & $\begin{array}{c}\text { Progressive right sided dystonia, symmetrical atrophy } \\
\text { and } \mathrm{T} 2 \text { hyperintensity of bilateral caudate nuclei and } \\
\text { putamina }\end{array}$ & $\mathrm{N}$ & 16 & 18 & Normal \\
\hline 13 & M / 21 & $\begin{array}{c}\text { Episodic abnormal involuntary movement since } 12 \\
\text { years old. }\end{array}$ & $\mathrm{N}$ & 14 & 19 & Normal \\
\hline
\end{tabular}

Footnotes: \#Triplet repeat primed PCR was performed for patients with one band detected and all showed normal results.
An overall prevalence from Europe, North American and Australia studies is about 5.7 per 100,000 based on eleven studies [7]. For Asia population, HD is much less common with an overall prevalence of 0.4 per 100,000 based on three studies values [8-10]. The different in size of CAG repeats and the frequency of HTT haplotypes may explain the different of geographic and ethnic difference of HD [7]. Clinical and genetic data in Chinese is limited, especially among Southern Chinese [5,8,11-18]. In this study, we report the clinical and genetic characteristics of six HD Hong Kong Chinese patients.

\section{Materials and Methods}

A total of thirteen referrals for HD genetic testing were performed during 2008 to 2013. Our laboratory is one of the two laboratories providing HD genetic test in the captioned period in Hong Kong. The clinical findings and number of CAG repeats found in each referral was summarized in Table 1. The test initiative was based on the clinical findings with the suspicion of HD or there were family members who had HD. A special consent form and information sheet was provided for HD genetic testing. Genetic counseling was performed by the referring neurologists and/or pathologists. For pre-symptomatic testing, collection of two EDTA blood specimens at different times was recommended. Otherwise, the whole analytical process was repeated by another operator. Patients were recommended to sign on the blood tube as well. 
PCR flanking the region containing CAG repeats sequence was performed. Primers for HD were used according to references [1924]. The primers and thermal cycling conditions were tabulated in Table 2. The $25 \mu \mathrm{L}$ reaction mixture which was the same for all primer sets contained 100 ng DNA template, 1x AmpliTaq Gold 360 Master Mix (Applied Biosystems), 5\% of GC enhancer (Applied BiTable 2: PCR primers and thermal cycling conditions. osystems), 400 pmol of each primer and DNAse free water. Positive control, normal DNA control and water blank were included in each analytical run. Before 2013, agarose gel electrophoresis was used for allele separation and size estimation by comparing with a DNA ladder marker, normal control and positive control (Figure 1a).

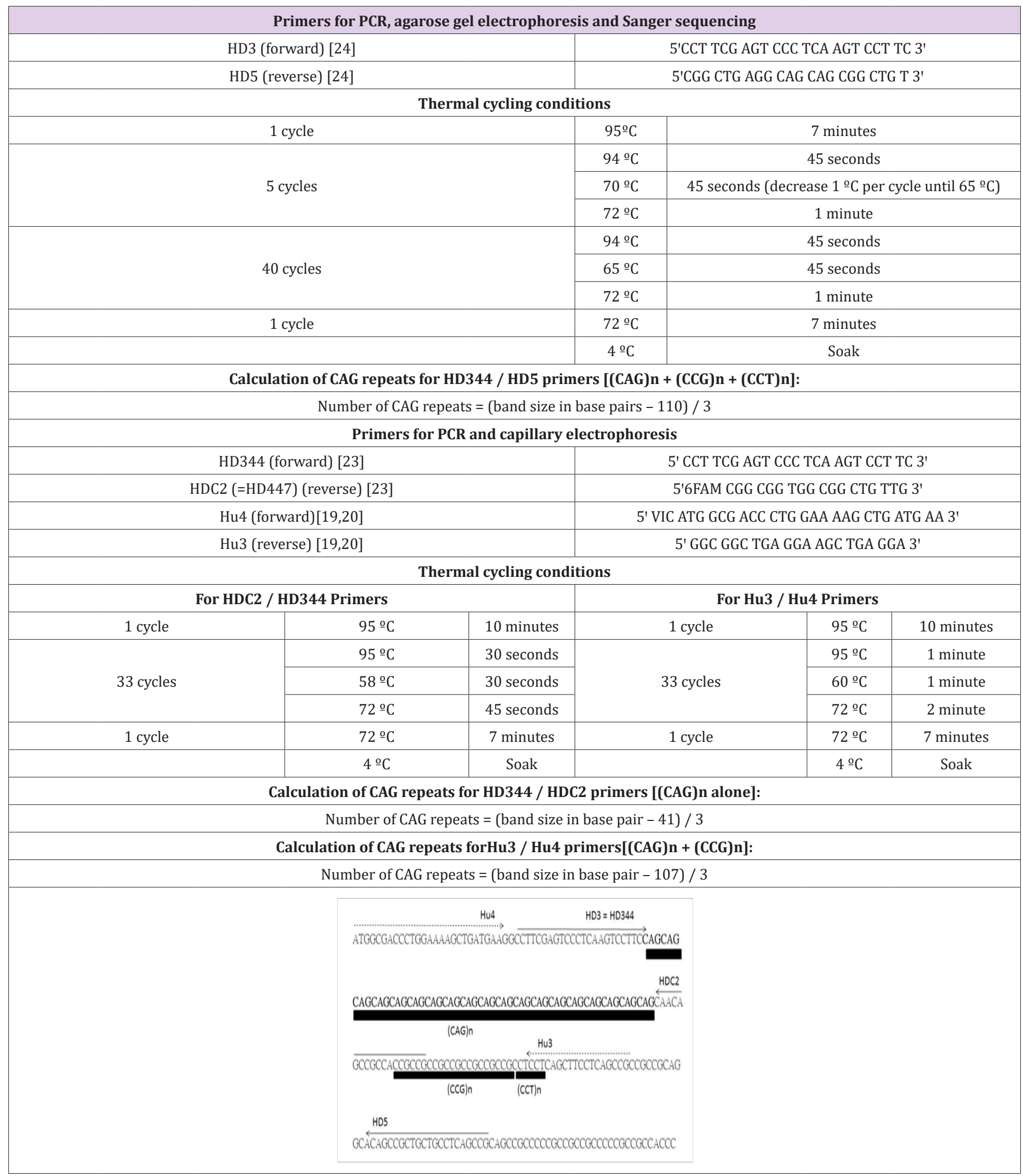




\begin{tabular}{|c|c|c|c|}
\hline \multicolumn{3}{|c|}{} \\
\hline \multirow{2}{*}{1 cycle } & $95{ }^{\circ} \mathrm{C}$ & 10 minutes & Podified thermal cycling condition of triplet repeat primed PCR \\
\hline \multirow{3}{*}{35 cycles } & $95{ }^{\circ} \mathrm{C}$ & 30 seconds & (forward) 5' 6FAM ATG AAG GCC TTC GAG TCC CTC AAG TCC 3' \\
\cline { 2 - 4 } & $64{ }^{\circ} \mathrm{C}$ & 1 minute & (reverse) 5' CGG TGG CGG CTG TTG CTG CTGCTGCTGCTG 3' \\
\cline { 2 - 4 } & $72{ }^{\circ} \mathrm{C}$ & 2 minutes & \\
\hline \multirow{2}{*}{1 cycle } & $72{ }^{\circ} \mathrm{C}$ & 15 minutes & \\
\hline
\end{tabular}

Band above $190 \mathrm{bp}$ (27 repeats), if present, would be excised from the agarose gel under low UV in the transilluminator for sequencing. All results were confirmed with Sanger sequencing. From 2013 onward, the agarose gel electrophoresis was replaced by capillary electrophoresis for allele detection and accurate sizing as it has better sensitivity and reproducibility. All positive or suspicious results were double-confirmed by Sanger sequencing (Figure 1b). Triplet repeat primed PCR was performed for cases with only one normal band detected using primers described by Jama et al. [25] Reflex genetic testing of DRPLA was performed for all negative cases except pre-symptomatic test with known HD family history. Practice guidelines on HD genetic testing were referenced [26-28]. Our laboratory participated in the European Molecular Genetics Quality Networks Huntington Scheme for regular monitoring the test accuracy of size detection with satisfactory performance. Our laboratory is accredited by the Hong Kong Laboratory Accreditation Scheme. Results were interpreted and reported independently by two senior staffs that at least one of them was a qualified pathologist who has obtained the Fellowship of the Hong Kong College of Pathologists in Chemical Pathology plus two years post fellowship professional experience including molecular genetics. Ethical approval has been obtained in local institute.

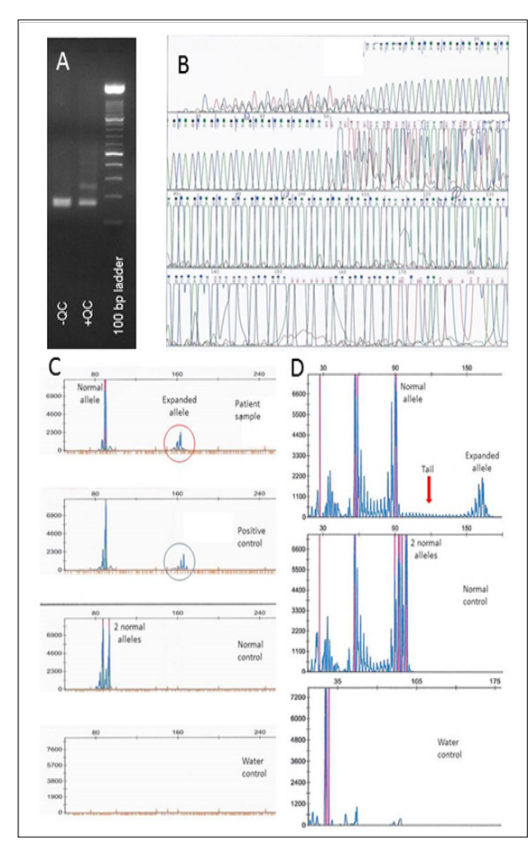

Figure 1.

\section{Results}

Out of the thirteen cases, six cases were diagnosed as HD (Table 1). Five positive cases were tested with clinical symptoms (Cases 2 ,
$3-5$ and 10) and the remained positive case was pre-symptomatic screening (Case 11). In addition, Case 6 also had a family history of HD but screened negative. All symptomatic HD patients were adult onset with $43.4 \pm 11.6$ years (mean \pm SD; range 29-59 years). The duration from onset to genetic testing was $7 \pm 4.8$ years (mean $\pm \mathrm{SD}$; range $0-12$ years). The major clinical presentations were generalized chorea, behavioral disorders, and dementia. Half of the symptomatic HD patients had no family history. For the three positive cases with family history, no evidence showed that the age of onset was much earlier than their affected parents. The number of pathological expanded CAG repeats was $46 \pm 6.8$ (range $42-58$ ). At the time of the last follow-up, none had died.

Two patients had carried out pre- $\neg$ testing after careful genetic counseling (Cases 6 and 11). Case 11 was positive for HD and her CAG repeats were more or less the same as her mother (42 versus 43 CAG repeats). The patient was calm to receive the result but she developed depressive symptoms related to other family problems on subsequent follow up which requiring anti-depressants. Though there is no sex prevalence for this autosomal dominant disease, all patients with diagnosed HD were female in our case series. Case 12 was an 8-year-old boy with progressive dystonia suspected of juvenile onset HD and two normal alleles were detected (16 and 18 CAG repeat sizes). In our case series, none of those with negative HD mutation were positive for DRPLA.

\section{Discussion}

HD is a progressive and fatal autosomal dominant neurodegenerative disorder with about 10 -fold lower prevalence among Asians. Our laboratory is one of the two major public laboratories providing HD genetic tests in Hong Kong whose population was 7.8 million. From 2008 to 2013, we only received 13 genetic test requests for suspected HD cases. Six of them were diagnosed having HD, of which three of them had no HD family history. The prevalence is lower than expected. On the other hand, chorea is the main clinical trigger for HD genetic test. However, since HD patients can present with Parkinsonism, dystonia and ataxia alone, we observed that the test is not frequently requested in such circumstances and this would probably lead to an under- or mis-diagnosis. We recommend reflex genetic testing for HD phenocopy conditions in patients with clinical symptoms of HD but tested negative HD mutation. In our case series, none of those with negative HD mutation were positive for DRPLA.

HD is a horrible disease with no cure and a grave prognosis. Patients should understand the risks, benefits and up-to-date limitations of the test results. Patient consent, pre- and post-test genetic counseling shall be performed by qualified professionals with proper molecular and clinical genetics training. Pre-symptomatic 
testing should be carefully considered as it involves significant psychological and emotional impacts. Wong et al. studied the psychosocial impact of HD on 28 asymptomatic subjects with family history of HD in Hong Kong Chinese [29]. One third showed depression and anxiety symptoms and one quarter would consider suicide if they screen positive. Good counseling can significantly alleviate this situation. We recommend that repeated counseling over a reasonable length of time is required. It is even more desirable if it is conducted by two independent counselors.

International Huntington Association and the World Federation of Neurology Research Group on Huntington's Chorea has issued guidelines for the genetics predictive testing in HD [30,31]. Pre-testing genetic counseling must be provided ensuring that the at-risk individual has a full understanding and make a knowledgeable decision about the risks and benefits of performing the test. In addition, two separate blood samples should be collected preferably at different time for pre-symptomatic testing. Report should be issued only if both samples results are consistent. Pre-symptomatic testing for minor below 18 years old is not recommended. Moreover, exclusion test should also be made available to couples facing reproductive choices along with prenatal diagnosis and preimplantation genetic diagnosis.

Juvenile HD is less common than adult onset HD with about a 10 -fold lower in prevalence. Jiang et al. reported a large case series of HD in 368 Chinese patients [17]. Among it, 6\% were childhood onset. Cao et al. also reported one of the six HD cases in two unrelated Chinese families was juvenile [32]. In contrast, there was no juvenile HD case documented in Hong Kong Chinese so far to our knowledge. Pediatric presentations vary differently from adult onset HD. It includes mainly, seizures, rigidity, dystonia and mental retardation rather than chorea. The clinical requests for such testing were also extremely low (only 1 in 5 years in this study). This likely reflects a low clinical suspicion index and subsequent under-diagnosis in our locality.

Technical method for HD genetic test has been well established since 1993. Primer sequences are still referenced from previously published literatures [19-24]. Specificity and sensitivity of the test are over $99 \%$. The accuracy of negative result also depends on the accuracy of the diagnosed HD family history. The presence of only one normal allele detected by conventional PCR-based methods cannot differentiate homozygous normal from compound heterozygous with one normal and one HD allele with very large expansion such as juvenile onset HD. Juvenile HD is usually in the range of 80 to 100 CAG repeat sizes. Gambardella et al. demonstrated a the successful detection of alleles carrying 115 CAG repeats by such method in a 9-year-old girl presented with 3-year history of progressive myoclonus epilepsy and negative family history [33]. On the other hand, Nance et al. showed that alleles carrying more than 125 CAG repeats cannot be reliably amplified [34]. Traditionally, such cases should be further tested by other methods such as Southern Blot analysis.

A recent paper with triplet repeat primed PCR for HD was published [25] and we successfully employed the primers into our mod- ified protocol (Table 2) and (Figure 1D). The $20 \mu \mathrm{L}$ reaction mixture contained 50 ng DNA template, 1x AmpliTaq Gold 360 Master Mix (Applied Biosystems), 4\% of GC enhancer (Applied Biosystems), 625 pmol of each primer and DNAse free water. Triplet repeat primed PCR is recommended to replace Southern blot for detection of very large expansions and confirmation of true homozygous normal genotypes [27]. Triplet repeat primed PCR results were normal in the three patients with one normal allele detected in our study. In addition, presence of polymorphism in HD gene may lead to poor primer annealing and thus amplification failure or allele drop-out. The frequency of polymorphisms in HD gene may be as great as 1 $\%$.

For expanded alleles, there may be presence of multiple bands on the gels. This may be due to combination of PCR artifact or lymphocytes mosaicism. In general, the most prominent band is used for reporting the number of repeats. On the other hand, direct comparison with other family member's alleles repeats number also help to resolve the problem. In our experience, we found that the repeat size can still be accurately measured by Sanger sequencing without prior gel excision of the expanded band procedure (Figure 1C). This could reduce the manual tediousness and avoid the uncertainty in choosing the less expanded band over the gel background with multiple bands. One of the many concerns among HD patients is whether their descendants would carry this disease gene. As most HDs are autosomal dominant, $50 \%$ of their descendants would have acquired this disease gene. In the past, they were advised to avoid conception, or half of their descendants will acquire HD. With the advance of in-vitro fertilisation and preimplantation genetic diagnosis, they can have normal babies who do not have this disease [35].

An understanding of HD genetics is critical to interpreting the results of HD genetic tests. Though testing of expanded CAG repeats is straight forward by PCR flanking the mutated gene exon, test limitation should be considered thoroughly before issuing the report. The report shall state the CAG repeat length of both alleles with the different CAG repeats categories and descriptors. But it should be noted that calculation of number of repeat at disease marginal range is limited by the method inaccuracy. In our method validation, the method is validated that the accuracy is \pm 1 CAG repeat for alleles less than 40 repeats and \pm 3 CAG repeats for higher CAG repeats. To minimize the possible error, samples from both parents and at least one affected family member should be available for result comparison.

\section{Conclusion}

HD is a progressive and fatal autosomal dominant neurodegenerative disorder typically with adult onset leading to motor, cognitive and emotional disturbances. We have characterized the clinical and genetic data of six Huntington disease Hong Kong Chinese patients. All were adult onset and had CAG repeat $>40$ and half had no HD family history. The duration from onset to genetic testing was significantly long. Clinical requests for HD genetic test are infrequent, especially juvenile, likely reflecting a low clinical suspicion index and subsequent under-diagnosis in our locality. We 
have simplified the PCR protocols of triplet repeat primed PCR and Sanger sequencing without gel excision for expanded allele. In our case series, none of those with negative HD mutation were positive for DRPLA. An understanding of HD genetics is critical to interpreting the results of HD genetic tests. We recommend reflex testing for other HD phenocopy conditions could be considered in patients with negative HD mutation.

\section{References}

1. Elliotson J (1832) Clinical lecture. Lancet 1: 161-67.

2. HuntingtonG (1872) On chorea, in The Medical and Surgical Reporter: A Weekly Journal SW Butler Philadelphia pp: 317-321.

3. (1993) A novel gene containing a trinucleotide repeat that is expanded and unstable on Huntington's disease chromosomes. The Huntington's Disease Collaborative Research Group. Cell 72(6): 971-983.

4. Sergio Alejandro Rodriguez-Quiroga, Dolores Gonzalez-Moron, Nelida Garretto, Marcelo Andres Kauffman2 (2013) Huntington's disease masquerading as spinocerebellar ataxia. BMJ Case Rep.

5. Y Dong, Y-M Sun, Z-J Liu, W Ni, S-S Shi, et al. (2013) Chinese patients with Huntington's disease initially presenting with spinocerebellar ataxia. Clin Genet 83(4): 380-383.

6. Squitieri F, Berardelli A, Nargi E, Castellotti B, Mariotti C, et al. (2000) Atypical movement disorders in the early stages of Huntington's disease: clinical and genetic analysis. Clin Genet 58(1): 50-56.

7. Pringsheim T, Wiltshire K, Day L, Dykeman J, Steeves T et al. (2012) The incidence and prevalence of Huntington's disease: a systematic review and meta-analysis. MovDisord 27(9): 1083-1091.

8. Chang CM, Yu YL, Fong KY, Wong MT, Chan YW et al. (1994) Huntington's disease in Hong Kong Chinese: epidemiology and clinical picture. Clin Exp Neurol 31: 43-51.

9. Chen YY, CH Lai (2010) Nationwide population-based epidemiologic study of Huntington's Disease in Taiwan. Neuroepidemiology 35(4): 250-254.

10. Nakashima K, Watanabe Y, Kusumi M, Nanba E, Maeoka Y, et al. (1996) Epidemiological and genetic studies of Huntington's disease in the Sanin area of Japan. Neuroepidemiology 15(3): 126-131.

11. CM Leung, Y W Chan, C M Chang, Y L Yu, C N Chen (1992) Huntington's disease in Chinese: a hypothesis of its origin. J NeurolNeurosurg Psychiatry 55(8): 681-684.

12. V Chan, Y L Yu, T P Chan, B Yip, C M Chang, et al. (1995) DNA analysis of Huntington's disease in southern Chinese. J Med Genet 32(2): 120-124.

13. Soong BW, JT Wang (1995) A study on Huntington's disease associated trinucleotide repeat within the Chinese population. ProcNatl Sci CouncRepub China B 19(3): 137-142.

14. Shan DE, Soong BW, Yeh SI, Cheng CH, Wu ZA et al. (1997) Genetic screening for Huntington's disease in Chinese patients with involuntary movements. Clin Neurol Neurosurg 99(4): 244-247.

15. Shi, Sheng-Shenga,b Lin, Yia Zhao, Gui-Xianb et al. (2012) A Chinese pedigree with an individual homozygous for CAG repeats of Huntington's disease. Psychiatr Genet 22(1): 53-54.

16. Hu Y, J Liang, S Yu (2014) High prevalence of diabetes mellitus in a fivegeneration Chinese family with Huntington's disease. J Alzheimers Dis 40(4): 863-868.

17. Jiang H, Sun YM, Hao Y, Yan YP, Chen K, Xin SH, et al. (2014) Huntingtin gene CAG repeat numbers in Chinese patients with Huntington's disease and controls. Eur J Neurol 21(4): 637-642.
18. Mingxia Yu, Xiaogai Li, Sanyun Wu, Ji Shen, Jiancheng Tu (2014) Examination of Huntington's disease in a Chinese family. Neural RegenRes 9(4): 440-446.

19. Riess O, Noerremoelle A, Soerensen SA, Epplen JT (1993) Improved PCR conditions for the stretch of (CAG)n repeats causing Huntington's disease. Hum Mol Genet 2(6): 637.

20. John M Valdes, Danilo A Tagle, Lawrence W Elmer, Francis S Collin (1993) A simple non-radioactive method for diagnosis of Huntington's disease. Hum Mol Genet 2(6): 633-634.

21. Warner JP, LH Barron, DJ Brock (1993) A new polymerase chain reaction (PCR) assay for the trinucleotide repeat that is unstable and expanded on Huntington's disease chromosomes. Mol Cell Probes 7(3): 235-239.

22. Andrew SE, Goldberg YP, Theilmann J, Zeisler J, Hayden MR. (1994) A CCG repeat polymorphism adjacent to the CAG repeat in the Huntington disease gene: implications for diagnostic accuracy and predictive testing. Hum Mol Genet 3(1): 65-67.

23. Y Paul Goldberg, Susan E Andrew, Lorne A Clarke, Michael R Hayden (1993) A PCR method for accurate assessment of trinucleotide repeat expansion in Huntington disease. Hum Mol Genet 2(6): 635-636.

24. Jama M, Millson A, Miller CE, Lyon E (2013) Triplet repeat primed PCR simplifies testing for Huntington disease. J Mol Diagn 15(2): 255-262.

25. Potter NT, EB Spector, TW Prior (2004) Technical standards and guidelines for Huntington diseasetesting. Genet Med 6(1): 61-65.

26. Bean L, P Bayrak-Toydemir (2014) American College of Medical Genetics and Genomics Standards and Guidelines for Clinical Genetics Laboratories, 2014 edition: technical standards and guidelines for Huntington disease. Genet Med 16(12): e2.

27. Monique Losekoot, Martine J van Belzen, Sara Seneca, Peter Bauer, Susan AR Stenhouse, et al. (2013) EMQN/CMGS best practice guidelines for the molecular genetic testing of Huntington disease. Eur J Hum Genet 21(5): 480-486.

28. Wong MT, Chang PC, Yu YL, Chan YW, Chan V (1994)Psychosocial impact of Huntington's disease on Hong Kong Chinese families. Acta PsychiatrScand 90(1): 16-18.

29. (1994) Guidelines for the molecular genetics predictive test in Huntington's disease. International Huntington Association (IHA) and the World Federation of Neurology (WFN) Research Group on Huntington's Chorea. Neurology 44(8): 1533-1536.

30. (1994) International Huntington Association and the World Federation of Neurology Research Group on Huntington's Chorea. Guidelines for the molecular genetics predictive test in Huntington's disease. J Med Genet 31(7): 555-559.

31. Cao GN, Bao XH, Lu HM, Zhang JJ, Ma YN et al. (2011) Clinical characteristics of Huntington disease in two pedigrees and analysis of expanded CAG trinucleotide repeat. Beijing Da XueXueBao 43(2): 163167.

32. Gambardella A, Muglia M, Labate A, Magariello A, Gabriele AL, et al. (2001) Juvenile Huntington's disease presenting as progressive myoclonic epilepsy. Neurology 57(4): 708-711.

33. Nance MA, Mathias-Hagen V, Breningstall G, Wick MJ, McGlennen RC (1999) Analysis of a very large trinucleotide repeat in a patient with juvenile Huntington's disease. Neurology 52(2): 392-394.

34. Lau KK, Ng EH, Tso YM, Mak CM (2015) Halt hereditary tragedy by bridging the gap between knowledge and application of technology. Hong Kong Med J 21: 188-190. 


\section{(C) (P) This work is licensed under Creative}

Submission Link: https://biomedres.us/submit-manuscript.php

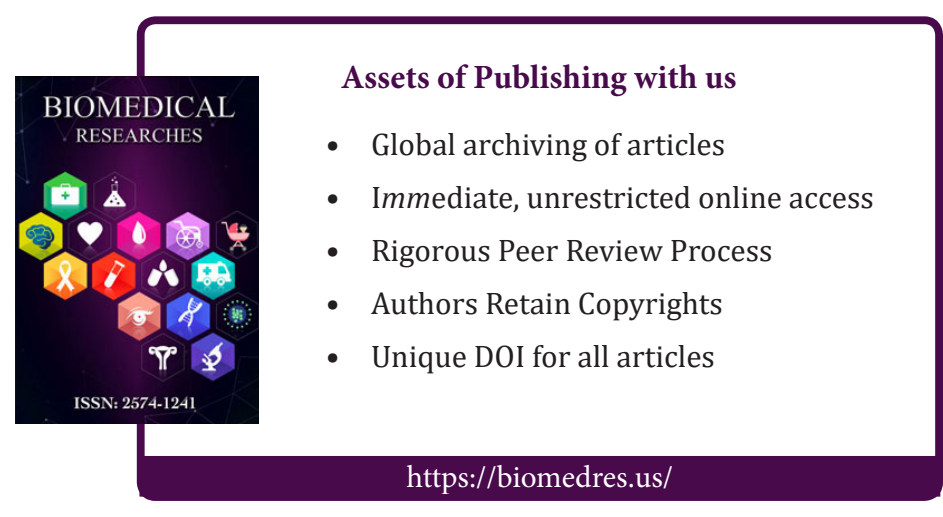

